\title{
Unified formulation of variational approaches and separable expansions for the solution of scattering equations
}

\author{
Sadhan K. Adhikari \\ Departamento de Física, Universidade Federal de Pernambuco, 50.000 Recife, Pernambuco, Brazil \\ Lauro Tomio \\ Instituto de Física Teórica, Universidade Estadual Paulista, 01.405 São Paulo, São Paulo, Brazil
}

(Received 9 March 1987)

\begin{abstract}
Both the variational approach and the method of separable expansion have proved to be very useful for the solution of scattering equations. Connections are established between these two approaches. It is shown that an underlying variational principle is responsible for the good convergence obtained with the method of separable expansions. It is pointed out that a proper choice of expansion functions is essential for the rapid convergence of the method of separable expansion. Several types of separable expansions are tested numerically for the Reid ${ }^{1} S_{0}$ soft-core potential. It is shown that with proper choices of expansion functions these separable expansions yield more rapid convergence than those obtained previously in other studies of separable expansions. In the case of the three-body scattering equations the usual separable expansions are shown to follow from the general expansion schemes studied here with special choices of expansion functions.
\end{abstract}

\section{INTRODUCTION}

Variational approaches ${ }^{1-7}$ have proved to be very useful for solving scattering equations which usually have the Lippmann-Schwinger ${ }^{8}$ (LS) form:

$$
T=V+V G_{0} T
$$

where $T$ is the transition $(t)$ matrix, $V$ the potential, and $G_{0}$ the free resolvent operator. There are different forms of variational principles which have been used in the solution of the LS equation. Similar variational principles have also been used in the solution of few-body equations of the Faddeev type. ${ }^{3}$ Here we classify the different variational principles in certain classes and also establish simple connections among some of them.

We classify the existing variational principles in essentially two classes: the Hulthen-Kohn-type ${ }^{2}$ and the Schwinger-type ${ }^{1}$ variational principles. A HulthénKohn-type variational principle is essentially a variational principle for the operator $T-V$. The simplest variational principle of this class is the usual $\mathrm{Kohn}^{2,3}$ variational principle. A Schwinger-type ${ }^{1,3}$ variational principle is, on the other hand, a variational principle for the transition matrix $T$. The simplest variational principle of this class is the usual Schwinger variational principle. Starting from the simplest variational principle in each class, one can generate complicated variational principles within each class simply by considering a more complicated variational function. This establishes a simple connection between variational principles within the same class. No such connection exists between a variational principle of the Hulthén-Kohn type and another of the Schwinger type.

The usual $\mathrm{Kohn}^{2}$ variational principle does not involve the resolvent operator and is the simplest of all the available variational principles. This variational principle, however, has the unpleasant feature of requiring trial functions having the same asymptotic behavior as the scattering wave functions. All variational principles of the Schwinger type and the more complicated variational principles of the Hulthen-Kohn type explicitly use the resolvent operator and can employ normalizable $L^{2}$ trial functions.

More complex classes of variational principles can be constructed for the operator $\left(T-V-V G_{0} V\right)$ using the idea of Schwinger and of Hulthén and Kohn. These will, in general, involve explicitly the resolvent operator $G_{0}$ and one can use normalizable trial functions.

Degenerate kernel schemes and methods of separable expansions have also been successfully used for the solution of scattering equations for both two- and threeparticle systems. These methods essentially rely upon approximating the kernel by a sum of separable terms. In different ways of the application of the method one generates a separable expansion for the operators $T$, $T-V$, or $T-V-V G_{0} V$, etc. Many such separable expansions have been suggested ${ }^{9-16}$ in the literature. Separable expansions for the two-body $t$ matrix $T$ are particularly very useful because of the simplicity they bring when used in the kernel of three body equations. ${ }^{17}$ The same is true for separable expansion of three body $t$ matrix in the context of the kernel of four body scattering equations. ${ }^{18}$

In an early study of the Schwinger and the HulthénKohn variational principles by Gerjuoy, Moe, and Saxon, ${ }^{4}$ the variational $t$ matrix was written essentially in a separable form. Later, for an appropriate choice of variational functions, equivalence was established ${ }^{7}$ between the Schwinger variational $t$ matrix and the usual separable expansion for the $t$ matrix. Similarly, we show that a Hulthén-Kohn-type variational principle leads to a se- 
parable expansion for the operator $T-V$.

Starting from a variational principle for $T-V$, Sloan and $B$ rady ${ }^{5}$ derived a separable expansion for $T-V$. We shall see that, though they called this variational principle one of the Schwinger type, the variational principle studied by them is intimately related to the usual Kohn variational principle and hence should be termed one of the Hulthén-Kohn type. They also deduced two separable expansions for the $t$ matrix which they termed nonvariational. We show that these two results essentially follow from the Schwinger-type variational principle.

In this paper we present a unified approach to the problem of separable expansions and show their relation to the variational principles. We emphasize that a correct choice of expansion functions is essential for a rapid convergence. We study in detail several types of separable expansions for the $t$ matrix using two types of form factors. The first type of form factor depends explicitly on the potential and the second type does not depend on the potential. It is pointed out that in the case of the three body $t$ matrix all the separable expansions proposed so far should be considered as special cases of separable expansions discussed in this paper.

In order to test how these expansions converge in practice, we performed numerical calculations in the case of four expansion schemes for the Reid ${ }^{1} S_{0}$ potential. ${ }^{19}$ We found that with appropriate choice of expansion functions each of these expansions converges faster than expansions studied before.

The plan of the paper is as follows. In Sec. II we present a new unified approach to the degenerate kernel method for LS equations and present various approximation schemes for the $t$ matrix. In Sec. III we establish relations between the degenerate kernel method of Sec. II and the variational principles of the Schwinger and the Hulthén-Kohn-type for operators $T$ and $T-V$, respectively. In Sec. IV a brief discussion of our approach is presented. In Sec. $\mathrm{V}$ we present numerical results for four of the approximation schemes of Sec. II for the Reid ${ }^{1} S_{0}$ potential. We end the paper with a brief summary in Sec. VI.

\section{DEGENERATE KERNEL APPROACHES}

In this paper we shall be limited to a discussion of the present approach for solving a LS-type equation written in the form

$$
T=V+V G_{0} T
$$

though many of the ideas presented in this paper are applicable in the case of a general Fredholm integral equation. In Eq. (2.1) $T$ is the $t$ matrix, $V$ the potential, and $G_{0}$ the resolvent operator defined by

$$
G_{0}=\left(E-H_{0}+i \epsilon\right)^{-1},
$$

where $H_{0}$ is the relative kinetic energy operator and $E+i \epsilon$ is the complex energy parameter with a small positive imaginary part $\epsilon \rightarrow 0$.

The present approach to the separable expansion is based on approximating the operator

$$
\mathcal{O}=A B^{-1} C,
$$

by the following rank $N$ approximation

$$
\mathcal{O}_{N}=\sum_{i, j=1}^{N} A\left|g_{i}\right\rangle D_{i j}\left\langle f_{j}\right| C,
$$

with

$$
\left(D^{-1}\right)_{j i}=\left\langle f_{j}|B| g_{i}\right\rangle .
$$

Here, $\left|g_{i}\right\rangle$ and $\left\langle f_{j}\right|$ are two sets of arbitrary chosen functions. The approximation (2.3) is independent of normalization of functions $\left|g_{i}\right\rangle$ and $\left\langle f_{j}\right|$. The approximation (2.3) is achieved from (2.2) by inserting a set of complete states

$$
\lim _{N \rightarrow \infty} \sum_{i=1}^{N}\left|g_{i}\right\rangle\left\langle g_{i}\right|
$$

between $A$ and $B^{-1}$ and

$$
\lim _{N \rightarrow \infty} \sum_{j=1}^{N}\left|f_{j}\right\rangle\left\langle f_{j}\right|
$$

between $\boldsymbol{B}^{-1}$ and $C$. If the functions $g_{i}$ and $f_{j}$ are assumed to form a complete set of states, then in the limit $N \rightarrow \infty$ in the space spanned by $f_{j}$ and $g_{i}$, $i, j=1,2, \ldots, N, \mathcal{O}$ of Eq. (2.2) can be taken to be identical to the approximation $\mathcal{O}_{N}$ of Eq. (2.3):

$$
\left\langle f_{j}|\mathcal{O}| g_{i}\right\rangle=\left\langle f_{j}\left|\mathcal{O}_{N}\right| g_{i}\right\rangle, \quad i, j=1,2, \ldots, N
$$

because we have the following assumed completeness relations:

$$
\begin{aligned}
1 & =\lim _{N \rightarrow \infty} \sum_{i=1}^{N}\left|g_{i}\right\rangle\left\langle g_{i}\right| \\
& =\lim _{N \rightarrow \infty} \sum_{j=1}^{N}\left|f_{j}\right\rangle\left\langle f_{j}\right|,
\end{aligned}
$$

which have been used to arrive at Eq. (2.3) from Eq. (2.2). When $N$ is large it is expected that $g_{i}$ and $f_{j}$ will span the relevant parts of the Hilbert space and $\mathcal{O}_{N}$ will be a good approximation to $\mathcal{O}$. In practice, however, one works with a large but finite $N$.

The above idea leads to various approximations for the $t$ matrix $T$. One can easily write $T$ of Eq. (2.1) in the forms

$$
\begin{aligned}
T & =V\left(V-V G_{0} V\right)^{-1} V \\
& =1\left(V^{-1}-G_{0}\right)^{-1} 1 \\
& =V\left(1-G_{0} V\right)^{-1} 1 \\
& =1\left(1-V G_{0}\right)^{-1} V
\end{aligned}
$$

etc. Using the above idea in Eq. (2.6), one has the expansion (with $f=g$ )

$$
T_{N}=\sum_{i, j=1}^{N} V\left|f_{i}\right\rangle D_{i j}\left\langle f_{j}\right| V,
$$

with

$$
\left(D^{-1}\right)_{j i}=\left\langle f_{j}\left|V-V G_{0} V\right| f_{i}\right\rangle,
$$


which is the approximation scheme of Ref. 7, where it has been used with good success. It has also been shown $^{7}$ that expansion (2.9) follows from the Schwinger variational principle. Similarly, Eq. (2.7) leads to

$$
\begin{aligned}
T_{N} & =\sum_{i, j=1}^{N}\left|g_{i}\right\rangle D_{i j}\left\langle g_{j}\right|, \\
\left(D^{-1}\right)_{j i}=B_{j i}-\left\langle g_{j}\left|G_{0}\right| g_{i}\right\rangle, & B_{j i}=\left\langle g_{j}\left|1 V^{-1} 1\right| g_{i}\right\rangle \\
& =\sum_{l, m=1}^{N}\left\langle g_{j} \mid f_{l}\right\rangle C_{l m}\left\langle f_{m} \mid g_{i}\right\rangle,
\end{aligned}
$$

where

$$
\left(C^{-1}\right)_{m l}=\left\langle f_{m}|V| f_{l}\right\rangle .
$$

Here the technique of Eqs. (2.2)-(2.4) has been applied twice; first, in relation to the operator $\left(V^{-1}-G_{0}\right)^{-1}$ and then in relation to $V^{-1}$ explicitly, and two types of expansion functions $f$ and $g$ are used in the two expansions. It is easy to verify that Eqs. (2.9) and (2.10) are solutions of the LS equation (2.1) with the following approximate potentials:

$$
V_{N}=\sum_{i, j}^{N} V\left|f_{i}\right\rangle J_{i j}\left\langle f_{j}\right| V
$$

and

$$
V_{N}=\sum_{i, j}^{N}\left|g_{i}\right\rangle K_{i j}\left\langle g_{j}\right|,
$$

respectively. Now $J$ and $K$ of Eqs. (2.11) and (2.12) are defined by

$$
\left(J^{-1}\right)_{j i}=\left\langle f_{j}|V| f_{i}\right\rangle
$$

and

$$
\left(K^{-1}\right)_{j i}=B_{j i}
$$

with $B$ given by $(2.10 \mathrm{c})$. The approximation schemes so defined are independent of the normalization of the arbitrary functions $f$ and $g$.

Finally, Eq. (2.8a) leads to the expansion

$$
T_{N}=\sum_{i, j=1}^{N} V\left|f_{i}\right\rangle D_{i j}\left\langle g_{j}\right| \text {, }
$$

with

$$
\left(D^{-1}\right)_{j i}=\left\langle g_{j}\left|\left(1-G_{0} V\right)\right| f_{i}\right\rangle,
$$

which is the simplest separable expansion that does not involve $V^{-1}$ or $G_{0}^{-1}$. Expansion (2.13) results if we solve the LS equation (2.1) with the approximate potential

$$
V_{N}=\sum_{i, j=1}^{N} V\left|f_{i}\right\rangle J_{i j}\left\langle g_{j}\right|,
$$

where

$$
\left(J^{-1}\right)_{j i}=\left\langle g_{j} \mid f_{i}\right\rangle .
$$

In this way it is possible to generate many approximation schemes. We shall mention finally one more expan- sion which results from

$$
T=V G_{0}\left(G_{0}-G_{0} V G_{0}\right)^{-1} .
$$

Using the method elaborated above we have

$$
T_{N}=\sum_{i, j=1}^{N} V G_{0}\left|g_{i}\right\rangle D_{i j}\left\langle g_{j}\right|,
$$

but now with

$$
\left(D^{-1}\right)_{j i}=\left\langle g_{j}\left|\left(G_{0}-G_{0} V G_{0}\right)\right| g_{i}\right\rangle .
$$

It is easy to realize that Eq. (2.15) follows from Eq. (2.13) by changing $\left|f_{i}\right\rangle$ to $G_{0}\left|g_{i}\right\rangle$. Expansion (2.15) was studied before by Sloan and Brady. ${ }^{5}$ Thus, by writing complicated expressions for $T$ or by replacing the expansion functions by more complex ones, one can derive many expansions for $T$. We shall see that all of them are intrinsically related to the Schwinger variational principle.

In a similar manner it is possible to make separable expansion for $T-V$ which is equivalent to writing $T$ as the sum of $V$ and a separable expansion. For example, applying the technique of $(2.2)-(2.4)$ to the last term of

$$
T=V+V(E-H+i \epsilon)^{-1} V,
$$

one gets the following approximate $t$ matrix,

$$
T_{N}=V+\sum_{i, j=1}^{N} V\left|f_{i}\right\rangle D_{i j}\left\langle\hat{f}_{j}\right| V,
$$

where

$$
\left(D^{-1}\right)_{j i}=\left\langle\hat{f}_{j}|(E-H)| f_{i}\right\rangle .
$$

Expansion (2.17) was obvious from the work of Takatsuka and McKoy. ${ }^{6}$ Equation (2.17) does not involve a resolvent operator and has the problems associated with the vanishing of the determinant of the matrix $D^{-1}$ in (spurious) positions other than bound states and resonances. Though this expansion is the simplest, it may not be very useful from a practical point of view. It has later been pointed out that very special care ${ }^{20}$ is needed to choose the expansion functions $f$ and $\hat{f}$. We shall show that expansion (2.17) is a consequence of the usual form of Kohn variational principle.

In the simplest choice, of course, one uses $f=\hat{f}$ in Eq. (2.17), though other choices are possible in which one can avoid the problem of spurious vanishing of the determinant of the matrix $D^{-1}$. One interesting choice is $\left\langle\hat{f}_{j}\right|=\left\langle g_{j}\right| G_{0}$, when Eq. (2.17) yields

$$
T_{N}=V+\sum_{i, j=1}^{N} V\left|f_{i}\right\rangle D_{i j}\left\langle g_{j}\right| G_{0} V,
$$

where

$$
\left(D^{-1}\right)_{j i}=\left\langle g_{j}\left|\left(1-G_{0} V\right)\right| f_{i}\right\rangle .
$$

Another possibility is to take $f=\hat{f}$ in Eq. (2.17) and replace $\left|f_{i}\right\rangle$ by $G_{0}\left|g_{i}\right\rangle$ and $\left\langle f_{j}\right|$ by $\left\langle g_{j}\right| G_{0}$, while Eq. (2.17) yields

$$
T_{N}=V+\sum_{i, j=1}^{N} V G_{0}\left|g_{i}\right\rangle D_{i j}\left\langle g_{j}\right| G_{0} V,
$$


where

$$
\left(D^{-1}\right)_{j i}=\left\langle g_{j}\left|\left(G_{0}-G_{0} V G_{0}\right)\right| g_{i}\right\rangle .
$$

Expansion (2.19) was studied by Sloan and Brady, ${ }^{5}$ who derived Eq. (2.19) from a variational principle which they termed as one of the Schwinger type. We do not agree with this conclusion of theirs for reasons we discuss in the next section. Now it is obvious that, by making special choices of $\left|f_{i}\right\rangle$ and $\left\langle\hat{f}_{j}\right|$ in Eq. (2.17), one gets a different expansion, which will be related to (2.17).

Finally, it is possible to make separable expansions for more complicated operators such as $T-V-V G_{0} V$. For example, applying the technique of $(2.2)-(2.4)$ to the last term of

$$
T=V+V G_{0} V+V G_{0}\left(V^{-1}-G_{0}\right)^{-1} G_{0} V
$$

one gets the following approximate $t$ matrix,

$T_{N}=V+V G_{0} V+\sum_{i, j=1}^{N} V G_{0}\left|f_{i}\right\rangle D_{i j}\left\langle g_{j}\right| G_{0} V$,

with

$$
\left(D^{-1}\right)_{j i}=\left\langle g_{j}\left|\left(V^{-1}-G_{0}\right)\right| f_{i}\right\rangle .
$$

Equation (2.21) is the simplest expansion of this type. In the next section we present a relation of the above expansions with variational principles.

\section{VARIATIONAL PRINCIPLES}

It has been shown in cases of some of the degenerate kernel approaches presented in the preceding section that they can be derived from some variational principles which are responsible for good convergences of these approaches. ${ }^{5,7}$ Some of the degenerate kernel schemes of the preceding section have been previously termed nonvariational ${ }^{5}$ and this has been made responsible for the poor convergence obtained with these schemes without making an adequate search for an appropriate expansion function. We shall show that all the degenerate kernel schemes presented in the preceding section can be derived from some variational principle, and the poor convergence rate obtained in previous numerical calculations using some of these schemes resulted because of the difficulty of finding an appropriate expansion function.

It has been shown in Ref. 7 that expansion (2.9) can be obtained from the usual Schwinger variational form for the $t$ matrix: ${ }^{1}$

$$
\begin{aligned}
\left\langle\mathbf{p}|T| \mathbf{p}^{\prime}\right\rangle= & \left\langle\mathbf{p}|\boldsymbol{V}| \psi_{\mathbf{p}^{\prime}}^{(+)}\right\rangle+\left\langle\psi_{\mathbf{p}}^{(-)}|\boldsymbol{V}| \mathbf{p}^{\prime}\right\rangle \\
& -\left\langle\psi_{\mathbf{p}}^{(-)}\left|\left(\boldsymbol{V}-\boldsymbol{V} \boldsymbol{G}_{0} \boldsymbol{V}\right)\right| \psi_{\mathbf{p}^{\prime}}^{(+)}\right\rangle,
\end{aligned}
$$

which is stationary with respect to small variations of $\left|\psi_{\mathrm{p}^{\prime}}^{(+)}\right\rangle$and $\left\langle\psi_{\mathrm{p}}^{(-)}\right|$around their correct values. The stationary property of this expression follows easily from the LS equations for $\psi_{\mathbf{p}^{\prime}}^{(+)}$and $\psi_{\mathbf{p}}^{(-)}$,

$$
\begin{aligned}
& \left|\psi_{\mathbf{p}^{\prime}}^{(+)}\right\rangle=\left|\mathbf{p}^{\prime}\right\rangle+G_{0} V\left|\psi_{\mathbf{p}^{\prime}}^{(+)}\right\rangle, \\
& \left\langle\psi_{\mathbf{p}}^{(-)}\right|=\langle\mathbf{p}|+\left\langle\psi_{\mathbf{p}}^{(-)}\right| V G_{0} .
\end{aligned}
$$

Using the following variational expressions for the wave functions in (3.1),

$$
\begin{aligned}
& \left|\psi_{\mathbf{p}^{\prime}}^{(+)}\right\rangle=\sum_{i=1}^{N} a_{i}\left(\mathbf{p}^{\prime}\right)\left|f_{i}\right\rangle, \\
& \left\langle\psi_{\mathbf{p}}^{(-)}\right|=\sum_{j=1}^{N} b_{j}(\mathbf{p})\left\langle f_{j}\right|,
\end{aligned}
$$

and demanding that expression (3.1) be stationary with respect to variations of $a_{i}\left(\mathbf{p}^{\prime}\right)$ and $b_{j}(\mathbf{p})$, one can solve for $a_{i}\left(\mathbf{p}^{\prime}\right)$ and $b_{j}(\mathbf{p})$ and, using Eqs. (3.1)-(3.3), one easily recovers ${ }^{7}$ expansion (2.9).

Expansion (2.10) is easily related to the Schwinger variational expansion (2.9). This can be seen by using $\left|g_{i}\right\rangle=V\left|f_{i}\right\rangle$ and $\left\langle f_{j}\right| V=\left\langle g_{j}\right|$ in Eqs. (2.10a) $-(2.10 \mathrm{c})$. In other words, Eq. (2.10) can be derived from the following stationary form for the $t$ matrix:

$$
\begin{aligned}
\left\langle\mathbf{p}|T| \mathbf{p}^{\prime}\right\rangle= & \left\langle\mathbf{p} \mid \chi_{\mathbf{p}^{\prime}}^{(+)}\right\rangle+\left\langle\chi_{\mathbf{p}}^{(-)} \mid \mathbf{p}^{\prime}\right\rangle \\
& -\left\langle\chi_{\mathbf{p}}^{(-)}\left|\left(V^{-1}-G_{0}\right)\right| \chi_{\mathbf{p}^{\prime}}^{(+)}\right\rangle,
\end{aligned}
$$

which is stationary with respect to small variations of $\left|\chi_{\mathrm{p}^{\prime}}^{(+)}\right\rangle$and $\left\langle\chi_{\mathrm{p}}^{(-)}\right|$around their correct values, and where

$$
\left|\chi_{\mathbf{p}^{\prime}}^{(+)}\right\rangle=V\left|\psi_{\mathbf{p}^{\prime}}^{(+)}\right\rangle \text {and }\left\langle\chi_{\mathrm{p}}^{(-)}\right|=\left\langle\psi_{\mathrm{p}}^{(-)}\right| V \text {. }
$$

The stationary property of (3.4) follows from the following equations satisfied by the $\chi$ 's:

$$
\begin{aligned}
& \left|\chi_{\mathbf{p}^{\prime}}^{(+)}\right\rangle=V\left|\mathbf{p}^{\prime}\right\rangle+V G_{0}\left|\chi_{\mathbf{p}^{\prime}}^{(+)}\right\rangle, \\
& \left\langle\chi_{\mathbf{p}}^{(-)}\right|=\langle\mathbf{p}| \boldsymbol{V}+\left\langle\chi_{\mathbf{p}}^{(-)}\right| G_{0} \boldsymbol{V} .
\end{aligned}
$$

Next, making the following variational expansions for the functions,

$$
\begin{aligned}
& \left|\chi_{\mathbf{p}^{\prime}}^{(+)}\right\rangle=\sum_{i=1}^{N} a_{i}\left(\mathbf{p}^{\prime}\right)\left|g_{i}\right\rangle, \\
& \left\langle\chi_{\mathbf{p}}^{(-)}\right|=\sum_{j=1}^{N}\left\langle g_{j}\right| b_{j}(\mathbf{p}),
\end{aligned}
$$

and demanding that expression (3.4) be stationary with respect to variations of coefficients $a_{i}\left(\mathbf{p}^{\prime}\right)$ and $b_{j}(\mathbf{p})$, one can easily solve for $a_{i}$ and $b_{j}$ and, using Eqs. (3.4)-(3.6), one recovers expansions $(2.10 \mathrm{a})-(2.10 \mathrm{c})$. Then technique of Eqs. (2.2)-(2.4) has again been applied to evaluate (2.10c). This has the advantage of making the result (2.10d) $-(2.10 \mathrm{e})$ independent of the normalization of the expansion functions. Expansion (2.10) has the advantage of having energy independent analytic simple form factors if the $f$ 's have that property, even if $V$ is energy dependent.

Next, we consider the separable expansion (2.13). It can easily be related to expansion (2.9) if one takes $\left\langle g_{j}\right|=\left\langle f_{j}\right| V$, when the two expansions become identical. Now it is easy to realize that expansion (2.13) should follow from the following stationary form for the $t$ matrix,

$$
\begin{aligned}
\left\langle\mathbf{p}|T| \mathbf{p}^{\prime}\right\rangle= & \left\langle\mathbf{p}|\boldsymbol{V}| \psi_{\mathbf{p}^{\prime}}^{(+)}\right\rangle+\left\langle\chi_{\mathbf{p}}^{(-)} \mid \mathbf{p}^{\prime}\right\rangle \\
& -\left\langle\chi_{\mathbf{p}}^{(-)}\left|\left(1-G_{0} \boldsymbol{V}\right)\right| \psi_{\mathbf{p}^{\prime}}^{(+)}\right\rangle,
\end{aligned}
$$


which is stationary with respect to small variations of $\psi_{\mathbf{p}^{\prime}}^{(+)}$and $\chi_{\mathbf{p}}^{(-)}$and where $\chi$ and $\psi$ are defined by $(3.5 \mathrm{~b})$ and $(3.2 \mathrm{a})$, respectively. The stationary property of (3.7) follows from Eqs. (3.2) and (3.5).

Though expansions (2.9), (2.10), and (2.13) all follow essentially from the Schwinger variational forms (3.1), (3.4), and (3.7) for the $t$ matrix, respectively, in practical numerical application the success of these expansions will depend on appropriate choice of expansion functions $f$ or $g$. Also, it is now easy to realize that expansion (2.15) can also be derived from a variational principle of the Schwinger type.

Next, we consider expansion (2.17), which is really a separable expansion for the operator $T-V$ rather than for $T$. This expansion can be obtained from the usual Kohn variational form for the $t$ matrix: ${ }^{2}$

$$
\begin{aligned}
\left\langle\mathbf{p}|T| \mathbf{p}^{\prime}\right\rangle= & \left\langle\mathbf{p}|\boldsymbol{V}| \mathbf{p}^{\prime}\right\rangle+\left\langle\mathbf{p}|\boldsymbol{V}| \hat{\chi}_{\mathbf{p}^{\prime}}^{(+)}\right\rangle \\
& +\left\langle\hat{\chi}_{\mathbf{p}}^{(-)}|\boldsymbol{V}| \mathbf{p}^{\prime}\right\rangle \\
& -\left\langle\hat{\chi}_{\mathbf{p}}^{(-)}\left|\left(G_{0}^{-1}-\boldsymbol{V}\right)\right| \hat{\chi}_{\mathbf{p}^{\prime}}^{(+)}\right\rangle,
\end{aligned}
$$

which is stationary with respect to small variations of $\hat{\chi}_{\mathbf{p}^{\prime}}^{(+)}$and $\hat{\chi}_{\mathrm{p}}^{(-)}$and where

$$
\begin{aligned}
& \left|\hat{\chi}_{\mathbf{p}^{\prime}}^{(+)}\right\rangle=\left|\psi_{\mathbf{p}^{\prime}}^{(+)}\right\rangle-\left|\mathbf{p}^{\prime}\right\rangle=G_{0}\left|\chi_{\mathbf{p}^{\prime}}^{(+)}\right\rangle, \\
& \left\langle\hat{\chi}_{\mathrm{p}}^{(-)}\right|=\left\langle\psi_{\mathbf{p}}^{(-)}\right|-\langle\mathbf{p}|=\left\langle\chi_{\mathrm{p}}^{(-)}\right| G_{0},
\end{aligned}
$$

with $\left|\chi_{\mathrm{p}^{\prime}}^{(+)}\right\rangle$and $\left\langle\chi_{\mathrm{p}}^{(-)}\right|$defined by Eq. (3.5). The stationary property of (3.8) follows from Eq. (3.5). Using the following variational expressions,

$$
\begin{aligned}
& \left|\hat{\chi}_{\mathbf{p}^{\prime}}^{(+)}\right\rangle=\sum_{i=1}^{N} a_{i}\left(\mathbf{p}^{\prime}\right)\left|f_{i}\right\rangle, \\
& \left\langle\hat{\chi}_{\mathbf{p}}^{(-)}\right|=\sum_{j=1}^{N} b_{j}(\mathbf{p})\left\langle\hat{f}_{j}\right|,
\end{aligned}
$$

in (3.8) and, demanding that (3.8) be stationary with respect to variations of $a_{i}\left(\mathbf{p}^{\prime}\right)$ and $b_{j}(\mathbf{p})$, one can solve for $a_{i}\left(\mathbf{p}^{\prime}\right)$ and $b_{j}(\mathbf{p})$ :

$$
\begin{aligned}
& a_{i}\left(\mathbf{p}^{\prime}\right)=\sum_{j=1}^{N} D_{i j}\left\langle\hat{f}_{j}|V| \mathbf{p}^{\prime}\right\rangle, \\
& b_{j}(\mathbf{p})=\sum_{i=1}^{N}\left\langle\mathbf{p}|V| f_{i}\right\rangle D_{i j},
\end{aligned}
$$

with $D$ given by $(2.17 \mathrm{~b})$ :

$$
\left(D^{-1}\right)_{j i}=\left\langle\hat{f}_{j}\left|\left(G_{0}^{-1}-V\right)\right| f_{i}\right\rangle=\left\langle\hat{f}_{j}|(E-H)| f_{i}\right\rangle \text {. }
$$

Substituting (3.10) in Eqs. (3.8) and (3.9), one recovers (2.17a).

In practical application of the Kohn method, the construction of the $D$ matrix via $(3.10 \mathrm{c})$ is a delicate task..$^{5,20}$ The matrix to be inverted involves $E-H$. For positive energies, $E-H$ has a continuous spectrum. So it can easily happen that one eigenvalue of the finite matrix is small, specially if the number of basis functions is large. Then matrix $D^{-1}$ becomes nearly singular, which causes special problem with expansion (2.17a). With all other expansions of Sec. II the matrix to be inverted becomes singular if $V G_{0}$ has an eigenvalue near 1 , which is the condition for a true bound state or resonance pole. This is why expansion (2.17a), although very instructive and simple, is not very useful from a practical point of view. ${ }^{5}$ For a numerical application of $(2.17 \mathrm{a})$ special care ${ }^{20}$ is needed for the choice of the expansion functions $f$ and $\widehat{f}$.

It is now easy to realize that expansion (2.18a) can be obtained from the following stationary expression for the $t$ matrix:

$$
\begin{aligned}
\left\langle\mathbf{p}|T| \mathbf{p}^{\prime}\right\rangle= & \left\langle\mathbf{p}|\boldsymbol{V}| \mathbf{p}^{\prime}\right\rangle+\left\langle\mathbf{p}|\boldsymbol{V}| \hat{\chi}_{\mathbf{p}^{\prime}}^{(+)}\right\rangle \\
& +\left\langle\chi_{\mathbf{p}}^{(-)}\left|G_{0} V\right| \mathbf{p}^{\prime}\right\rangle \\
& -\left\langle\chi_{\mathbf{p}}^{(-)}\left|\left(1-G_{0} V\right)\right| \hat{\chi}_{\mathbf{p}^{\prime}}^{(+)}\right\rangle,
\end{aligned}
$$

where $\left|\hat{\chi}_{\mathbf{p}^{\prime}}^{(+)}\right\rangle$and $\left\langle\chi_{\mathrm{p}}^{(-)}\right|$are defined by Eqs. (3.5) and (3.9). Equation (3.11) is again a stationary expression for $T-V$, rather than for $T$. The stationary property follows from the use of Eqs. (3.5) and (3.9). Using the following variational expressions,

$$
\begin{aligned}
& \left|\hat{\chi}_{\mathbf{p}^{\prime}}^{(+)}\right\rangle=\sum_{i=1}^{N} a_{i}\left(\mathbf{p}^{\prime}\right)\left|f_{i}\right\rangle, \\
& \left\langle\chi_{\mathbf{p}}^{(-)}\right|=\sum_{j=1}^{N} b_{j}(\mathbf{p})\left\langle g_{j}\right|,
\end{aligned}
$$

in (3.11) and, demanding that (3.11) be stationary with respect to variations of $a_{i}\left(\mathbf{p}^{\prime}\right)$ and $b_{j}(\mathrm{p})$, one can obtain expansion (2.18a).

Finally, expansion (2.19a) can be easily shown, in a similar way, to follow from the following variational estimate for the $t$ matrix:

$$
\begin{aligned}
\left\langle\mathbf{p}|T| \mathbf{p}^{\prime}\right\rangle= & \left\langle\mathbf{p}|V| \mathbf{p}^{\prime}\right\rangle+\left\langle\mathbf{p}\left|V G_{0}\right| \chi_{\mathbf{p}^{\prime}}^{(+)}\right\rangle \\
& +\left\langle\chi_{\mathbf{p}}^{(-)}\left|G_{0} V\right| \mathbf{p}^{\prime}\right\rangle \\
& -\left\langle\chi_{\mathbf{p}}^{(-)}\left|\left(G_{0}-G_{0} V G_{0}\right)\right| \chi_{\mathbf{p}^{\prime}}^{(+)}\right\rangle .
\end{aligned}
$$

This variational principle was used by Sloan and $\mathrm{Brady}^{5}$ to derive expansion (2.19a); as a by-product they also derived two other approximations:

$$
\begin{aligned}
& T_{N}=\sum_{i, j=1}^{N}\left|g_{i}\right\rangle D_{i j}\left\langle g_{j}\right| G_{0} V, \\
& T_{N}=\sum_{i, j=1}^{N} V G_{0}\left|g_{i}\right\rangle D_{i j}\left\langle g_{j}\right|,
\end{aligned}
$$

which they termed nonvariational. Expansion (3.14b) is essentially the same as expansion ( $2.15 \mathrm{a})$. It is now clear that expansions (2.15a) or (3.14) can be derived from a Schwinger-type variational principle.

The stationary expression (3.13) for the $t$ matrix gives essentially a stationary estimate for the operator $T-V$ and hence is easily related to the Kohn variational form (3.8). So, variational principle (3.13) should be termed a Hulthén-Kohn-type variational principle and not a Schwinger-type variational principle as suggested by Sloan and Brady. ${ }^{5}$ Expansions (2.17)-(2.19) are easily related with each other if we make special choices for 
the expansion functions in (2.17), and all of them essentially follow from the Kohn variational principle. Similarly, expansions (2.9), (2.10), (2.13), and (2.15) can be easily related with each other if one makes special choices of expansion functions. All these latter expansions essentially follow from the Schwinger variational principle, but no such relation can be established between an expansion of the former type and an expansion of the latter type.

Now it is not difficult to realize that expansion (2.20) follows from the following stationary expression for the $t$ matrix:

$$
\begin{aligned}
\left\langle\mathbf{p}|T| \mathbf{p}^{\prime}\right\rangle= & \left\langle\mathbf{p}\left|\left(V+V G_{0} V\right)\right| \mathbf{p}^{\prime}\right\rangle \\
& +\left\langle\mathbf{p}\left|V G_{0}\right| \widehat{\psi}_{\mathbf{p}^{\prime}}^{(+)}\right\rangle+\left\langle\hat{\psi}_{\mathbf{p}}^{(-)}\left|G_{0} V\right| \mathbf{p}^{\prime}\right\rangle \\
& -\left\langle\hat{\psi}_{\mathbf{p}}^{(-)}\left|\left(V^{-1}-G_{0}\right)\right| \hat{\psi}_{\mathbf{p}^{\prime}}^{(+)}\right\rangle,
\end{aligned}
$$

where

$$
\left|\hat{\psi}_{\mathbf{p}^{\prime}}^{(+)}\right\rangle=V G_{0} V\left|\psi_{\mathbf{p}^{\prime}}^{(+)}\right\rangle
$$

and

$$
\left\langle\widehat{\psi}_{\mathbf{p}}^{(-)}\right|=\left\langle\psi_{\mathbf{p}}^{(-)}\right| V G_{0} V .
$$

Variational principle $(3.15)$ is really a variational principle for the operator $T-V-V G_{0} V$ and hence is neither one of the Hulthén-Kohn nor one of the Schwinger type.

From the discussion of this section it is obvious that one can always associate a variational principle with a degenerate kernel scheme for the $t$ matrix. This will be true, in general, and one can construct other degenerate kernel schemes for the $t$ matrix and find out the underlying variational principle from which it is derived.

\section{DISCUSSION}

The methods presented in Secs. II and III not only give a unified degenerate kernel approach to the solution of the LS equation, but will have application in more complex multichannel few-body problems, especially in making separable expansions for the three-body $t$ matrix. Many of the approaches have already been used in various contexts. Also, many approaches of other authors can easily be related to the methods presented here with special choices of expansion functions. In this section we relate our approaches to those of other authors.

Essentially, the simplest Kohn variational expression (2.17a) has been recently used by Rawitscher and Delic ${ }^{15}$ in the special case where $f_{i}=\widehat{f}_{i}=\phi_{i}$. They used the energy dependent Bessel-Weinberg states $\phi_{i}$ with the property $^{15}$

$$
\left\langle\phi_{i}\left|\left(E-H_{0}\right)\right| \phi_{j}\right\rangle=\delta_{i j},
$$

where $(2.17 \mathrm{~b})$ simplifies to

$$
\left(D^{-1}\right)_{j i}=\left\langle\phi_{j}|(1-V)| \phi_{i}\right\rangle .
$$

However, they failed to note the relation of this approach to the Kohn variational principle. With the Bessel-Weinberg states the numerical problem with the inversion of matrix $D^{-1}$ was avoided. We do not see much advantage in their approach, as they have to rely on numerically constructed energy dependent functions, in general, which did not yield impressive convergence for the Woods-Saxon model studied by them-for the $S$ wave case 15 expansion functions were needed to obtain results correct to three significant figures. (The WoodsSaxon potential studied by them is expected to converge faster than the soft core Reid potential studied by other authors.)

The method proposed by Révai, Sotona, and Žofka ${ }^{14}$ bears some similarity with the method of Eqs. (2.10). Specifically, they employ $g_{i}=f_{i}$ in (2.10) and approximate the coefficients $\left\langle f_{j} \mid f_{l}\right\rangle$ by certain weight functions:

$$
\left\langle f_{j} \mid f_{l}\right\rangle=\sigma_{j}^{N} \delta_{j l} .
$$

The functions $f$ are the harmonic oscillator basis functions. The convergence obtained by them is rather slow in the case of the Reid ${ }^{1} S_{0}$ potential-some 20 expansion terms are needed to get a result accurate to two significant figures. In the case of the Woods-Saxon potential, which is usually expected to show better convergence properties, their convergence is reasonably impressive. From this it is expected that the method of Rawitscher and Delic, ${ }^{15}$ if applied to the case of the Reid ${ }^{1} S_{0}$ potential, will show poorer convergence properties than those obtained in their treatment of the Woods-Saxon potential. In our numerical study with expansion (2.10), we shall see that if the expansion functions are properly chosen, it can show good convergence properties, and the poor convergence obtained by Révai et $a l .{ }^{14}$ is related to their use of inappropriate expansion functions.

Sloan and Brady ${ }^{5}$ studied expansion (2.15a) and did not obtain satisfactory convergence. We believe an inappropriate choice of expansion function was responsible for the unsatisfactory convergence obtained by them.

Much effort ${ }^{6}$ has been recently made to relate Hulthén-Kohn - and Schwinger-type variational principles, and also to find out which of these two types of variational principles converges faster. Though answers were given to this question in limited contexts by different workers, we do not believe that an absolute answer to this question even should exist. This is because we have seen that these two types of variational principles are really stationary expressions for two different operators $-T$ and $T-V$ for the Schwingerand the Hulthén-Kohn-type variational principles, respectively. So, in a general context one should not try to relate the two types of variational principles. Also, the success of these separable expansions should depend on an appropriate choice of expansion functions. As we are expanding two different functions in these two approaches, it is expected that the expansion functions should be different in these two cases. The expansion functions, which give good results in the context of the Schwinger variational principle, should and may not yield good result in the case of the Kohn variational principle. Hence the calculation of Takatsuka, Lucchese, and McKoy, ${ }^{6}$ who demonstrate the better convergence properties of the Schwinger variational principle over the Kohn variational principle while using same ex- 
pansion functions in both approaches in a simple model potential, do not and should not give any general answer to the question of the superiority of one type of variational principle over another, because the conclusions of Ref. 6 are expected to be model dependent.

It is interesting to note at this stage that, with a special choice of expansion function, expansion (2.9) yields most of the commonly used separable expansions. The Weinberg series ${ }^{9}$ is readily obtained if we take

$$
\boldsymbol{V}\left|f_{i}\right\rangle=\left|\psi_{i}\right\rangle,\left\langle f_{j}\right| \boldsymbol{V}=\left\langle\bar{\psi}_{j}\right|
$$

in Eq. (2.9), where the $\psi$ 's are (energy-dependent) eigenfunctions of the LS kernel, satisfying

$$
\begin{aligned}
& \lambda_{i} V G_{0}\left|\psi_{i}\right\rangle=\left|\psi_{i}\right\rangle, \\
& \lambda_{j}\left\langle\bar{\psi}_{j}\right| G_{0} V=\left\langle\bar{\psi}_{j}\right| .
\end{aligned}
$$

The biorthogonality relation

$$
\left\langle\bar{\psi}_{j}\left|G_{0}\right| \psi_{i}\right\rangle=\delta_{i j}\left\langle\bar{\psi}_{i}\left|G_{0}\right| \psi_{i}\right\rangle
$$

reduces (2.9) to

$$
T_{N}=\sum_{i=1}^{N} \frac{\left|\psi_{i}\right\rangle\left\langle\bar{\psi}_{i}\right|}{\left(\lambda_{i}-1\right)\left\langle\bar{\psi}_{i}\left|G_{0}\right| \psi_{i}\right\rangle} .
$$

Weinberg series (4.4) uses energy dependent expansion functions $\psi$.

The unitary pole expansion (UPE) uses the eigenfunctions $\psi$ of Eq. (4.3) at a bound state energy - B - and then Eq. (2.9) reduces to ${ }^{9}$

$$
\begin{aligned}
& T_{N}=\sum_{i, j=1}^{N}\left|\psi_{i}(-B)\right\rangle D_{i j}\left\langle\bar{\psi}_{j}(-B)\right|, \\
& \left(D^{-1}\right)_{j i}=\lambda_{i}(-B) \delta_{j i}-\left\langle\bar{\psi}_{j}(-B)\left|G_{0}\right| \psi_{i}(-B)\right\rangle .
\end{aligned}
$$

With appropriate choices of expansion functions Eq. (2.9) has been shown ${ }^{7,11}$ to yield the expansions ${ }^{10}$ of Ernst, Shakin, and Thaler, and of Bateman.

The UPE (4.5) is very useful because there the expansion is done via energy independent form factors. The UPE of (4.5) is not readily generalized to the case of the $t$ matrix involving more than two particles.

The main problem with the three particle system is that though in this case one has a LS-type equation, the potential appearing in this equation is energy dependent. It is easy to realize now that $T$ of Eq. (4.5a) can be obtained if we solve the LS equation with the following separable expansion for $V$ :

$$
V_{N}=\sum_{i=1}^{N}\left|\psi_{i}(-B)\right\rangle \frac{1}{\lambda_{i}(-B)}\left\langle\bar{\psi}_{i}(-B)\right|
$$

As $V$ is energy dependent in the case of the threeparticle system, the energy independent approximation (4.6) must fail at energies $E \neq-B$. Hence a modification of the UPE is necessary. One modification of the UPE has been proposed by Casel, Haberzettl, and Sandhas, ${ }^{18}$ known as the generalized UPE (GUPE). In this case the
LS equation is schematically written as

$$
T=V(E)+V(E) G_{0}(E) T,
$$

where the energy dependence of $T$ is not explicitly shown. We show that the GUPE follows with a special choice of expansion function in Eq. (2.10), which provides us with a simple way of making a separable approximation for the $t$ matrix with energy independent form factors, if the functions $g$ are energy independent.

Here we have expansion functions $\psi$ satisfying

$$
\begin{aligned}
& \lambda_{i} V(-B) G_{0}(-B)\left|\psi_{i}(-B)\right\rangle=\left|\psi_{i}(-B)\right\rangle, \\
& \lambda_{j}\left\langle\bar{\psi}_{j}(-B)\right| G_{0}(-B) V(-B)=\left\langle\bar{\psi}_{j}(-B)\right|,
\end{aligned}
$$

and normalized according to

$$
\left\langle\bar{\psi}_{j}(-B)\left|G_{0}(-B)\right| \psi_{i}(-B)\right\rangle=-\delta_{i j} .
$$

Now, in Eq. (2.10), if we take

$$
\begin{aligned}
& \left|g_{i}\right\rangle=\left|\psi_{i}(-B)\right\rangle, \quad\left\langle g_{j}\right|=\left\langle\bar{\psi}_{j}(-B)\right|, \\
& \left|f_{l}\right\rangle=G_{0}(-B)\left|\psi_{l}(-B)\right\rangle, \\
& \left\langle f_{m}\right|=\left\langle\bar{\psi}_{m}(-B)\right| G_{0}(-B),
\end{aligned}
$$

the use of (4.8) reduces (2.10) to

$$
T_{N}=\sum_{i, j=1}^{N}\left|\psi_{i}(-B)\right\rangle D_{i j}\left\langle\bar{\psi}_{j}(-B)\right|,
$$

where

$$
\left(D^{-1}\right)_{j i}=B_{j i}-\left\langle\bar{\psi}_{j}(-B)\left|G_{0}(E)\right| \psi_{i}(-B)\right\rangle
$$

and

$$
\left(B^{-1}\right)_{j i}=\left\langle\bar{\psi}_{j}(-B)\left|G_{0}(-B) V(E) G_{0}(-B)\right| \psi_{i}(-B)\right\rangle .
$$

Equation (4.10) constitutes the GUPE of Casel, Haberzettl, and Sandhas. ${ }^{18}$ If $V$ is energy independent, Eq. (4.10) reduces to the usual UPE (4.5). The present derivation of the GUPE explicitly demonstrates the fact that it follows from a special choice of expansion function in a more general approximation scheme-Eqs. (2.10) - considered in this paper. The relation of (2.10) to the Schwinger variational principle as demonstrated in Sec. III also explicitly shows that the GUPE should follow from a special choice of expansion function in a Schwinger-type variational principle - Eq. (3.4) - for the $t$ matrix.

So the formalism presented in this paper has interesting application in the case of the three-body problem. Another alternative to the GUPE in this case is the energy dependent pole expansion (EDPE), which was constructed by Sofianos, McGurk, and Fiedeldey ${ }^{18}$ using expansion (2.9). Now it is also easy to realize that two more expansions proposed by Fonseca, Haberzettl, and Cravo $^{18}$ follow also from expansion (2.9) with a special choice of expansion function, which has never been explicitly pointed out in the literature. All these demonstrate the usefulness and generality of the formalism presented in this paper. 


\section{NUMERICAL CALCULATIONS}

In order to see how the methods presented in this paper work in practice, we have performed numerical calculations with the Reid ${ }^{19}{ }^{1} S_{0}$ soft core potential, which is highly repulsive at short distances, and this makes it particularly difficult to obtain good convergence with this potential. So, if we can demonstrate good convergence with this potential, it is expected that the method will converge well with other potentials. This potential is constructed as a linear superposition of various $\mathrm{Yu}$ kawas and in momentum space it is 5,19

$$
V_{0}\left(p, p^{\prime}\right)=\frac{1}{4 \beta_{1} p p^{\prime}} \sum_{i=1}^{3} v_{i} \ln \left(\frac{\left(p+p^{\prime}\right)^{2}+\beta_{i}^{2}}{\left(p-p^{\prime}\right)^{2}+\beta_{i}^{2}}\right),
$$

where $\beta_{1}=0.7 \mathrm{fm}, \beta_{2}=4 \beta_{1}, \beta_{3}=7 \beta_{1}, v_{1}=-10.463 \mathrm{MeV}$, $v_{2}=-1650.6 \mathrm{MeV}$, and $v_{3}=6484.2 \mathrm{MeV}$.

We chose to study only four approximation schemes presented in this paper, namely Eqs. (2.9), (2.10), (2.13), and (2.18). We choose to study these expansions numerically, not because we think them to be superior to the other expansions in a general context, but because they are reasonably simple and will allow us to make some general conclusions about the convergence of these methods. Specifically, Eq. (2.9) was studied ${ }^{7}$ before and was shown to produce good convergence. We shall demonstrate that if the expansion functions are appropriately chosen one can significantly improve on the convergence rate obtained before. Equation (2.10) forms the basis of the GUPE. A previous application of Eq. (2.10) in the context of the Reid ${ }^{1} S_{0}$ potential produced poor convergence. ${ }^{14}$ We show that though numerical application of Eq. (2.10) is more complicated, in general, because it involves inversion of two matrices, good convergence can be obtained with the proper choice of expansion function. Equations (2.13) and (2.18) use nonsymmetric approximations for the $t$ matrix. We shall demonstrate that numerically this does not cause any special problem and rapid convergence can be obtained with an appropriate choice of expansion function.

We shall use simple analytic normalizable $L^{2}$ functions as expansion functions in these cases. As we do a momentum space calculation for the $t$ matrix, we would like to build in appropriate asymptotic properties in these expansion functions in the momentum space. We would like to construct the expansion functions using the following functions, ${ }^{21}$

$F_{n}(p)=\frac{1}{p^{2}+\beta^{2}} C_{n-1}^{1}\left(\frac{p^{2}-\beta^{2}}{p^{2}+\beta^{2}}\right), \quad n=1,2, \ldots, N$

which have been successfully used in similar calculations. ${ }^{5,7}$ In Eq. (5.2) $C_{n-1}^{1}$ is the Gegenbauer polynomi$\mathrm{al}^{21}$ of degree $n-1$, where $\beta$ is an arbitrary parameter. The set (5.2) is formally equivalent to the set of functions $\left(p^{2}+\beta^{2}\right)^{-n}, n=1, \ldots, N$. The functions defined by (5.2) are actually Sturmian functions for the Coulomb potential and hence are orthogonal with respect to certain weight functions. ${ }^{5}$ Because of this orthogonality property the matrices to be inverted in the present calculations become well conditioned.
First, let us consider expansion (2.9). It is easy to realize that this expansion will converge well if the $p$ dependence of $\langle q|T| p\rangle$ is well represented by the $p$ dependence of $\left\langle f_{j}|V| p\right\rangle$, or equivalently, if the $p$ dependence of $\langle q|V| p\rangle$ is well represented by the $p$ dependence of $\left\langle f_{j}|\boldsymbol{V}| p\right\rangle$. For small $p,\langle q|\boldsymbol{V}| p\rangle$ tends to a constant independent of $p$ and, as $p \rightarrow \infty$, $\langle q|V| p\rangle$ behaves like a polynomial in $p^{-2}$ with the leading term $\sim p^{-2}$, when the potential $V$ is given by (5.1). The $p$ dependence of $\left\langle f_{j}|V| p\right\rangle$ can be obtained from the following explicit forms,

$$
\left\langle f_{j}|V| p\right\rangle=\int_{0}^{\infty} j_{0}(p r) V(r) b_{j}(r) r^{2} d r,
$$

where

$$
b_{j}(r)=\left(\frac{2}{\pi}\right)^{1 / 2} \int_{0}^{\infty} j_{0}(p r) f_{j}(p) p^{2} d p
$$

with $f_{j}(p)=\left\langle f_{j} \mid p\right\rangle$. It is straightforward to verify, by using Eq. (5.3), that if we take

$$
f_{n}(p)=p^{-2}\left(p^{2}+\beta^{2}\right)^{-n}, \quad n=1, \ldots, N
$$

then $\left\langle f_{j}|V| p\right\rangle$ has the desired $p$ dependence: In the case of a Yukawa potential, we note the following.

(i) As $p \rightarrow 0,\left\langle f_{j}|V| p\right\rangle$ tends to a constant.

(ii) As $p \rightarrow \infty,\left\langle f_{j}|\boldsymbol{V}| p\right\rangle$ behaves like a polynomial in $p^{-2}$ with the leading term $\sim p^{-2}$. Recalling ${ }^{5}$ that the functions of Eq. (5.2) are formally equivalent to the functions $\left(p^{2}+\beta^{2}\right)^{-n}, n=1, \ldots, N$, we use the following set of functions in our numerical study of (2.9) in place of the set (5.4):

$$
\begin{aligned}
f_{n}(p) & \equiv p^{-2} F_{n}(p) \\
& =\frac{1}{p^{2}} \frac{1}{p^{2}+\beta^{2}} C_{n-1}^{1}\left(\frac{p^{2}-\beta^{2}}{p^{2}+\beta^{2}}\right), n=1, \ldots, N .
\end{aligned}
$$

The functions (5.5) are orthogonal with respect to certain weight functions and hence have the advantage of leading to a well conditioned matrix $D^{-1}$ of Eq. (2.9), which is to be inverted numerically.

Next we consider expansion (2.10). Here we have two sets of expansion functions $f$ and $g$. It is easy to realize that the functions $g$ should be so chosen that $\left\langle g_{i} \mid p\right\rangle$ represents well the $p$ dependence of $\langle q|T| p\rangle$ or, equivalently, of $\langle q|V| p\rangle$, namely, $\left\langle g_{i} \mid p\right\rangle$ should tend to a constant as $p \rightarrow 0$ and should behave like a polynomial in $p^{-2}$ with the leading term $p^{-2}$ as $p \rightarrow \infty$ with $V$ given by (5.1). It is easy to realize that functions (5.2) satisfy this property. Hence we take

$$
g_{i}(p) \equiv\left\langle g_{i} \mid p\right\rangle=F_{i}(p),
$$

where $F_{i}(p)$ is given by Eq. (5.2). In Eqs. (2.10) $f$ 's enter in the following expansion,

$$
V_{N}^{-1}=\sum_{l, m=1}^{N}\left|f_{l}\right\rangle C_{l m}\left\langle f_{m}\right|
$$

with 


$$
\left(C^{-1}\right)_{m l}=\left\langle f_{m}|V| f_{l}\right\rangle .
$$

In the limit $N \rightarrow \infty, V_{N}^{-1}$ of $(5.7 \mathrm{a}) \rightarrow V^{-1}$ in the space spanned by functions $f$; and in this limit, by multiplying Eq. (5.7a) by $V$ from the right and from the left, one arrives at

$$
V_{N}=\sum_{l, m=1}^{N} V\left|f_{l}\right\rangle C_{l m}\left\langle f_{m}\right| V .
$$

As Eqs. (5.7a) and (5.8) are equivalent, the functions $f$, which are good in the context of expansion (5.8), will also be good in the context of expansion (5.7a). As expansion (2.9) results if we solve the LS equation with the separable expansion (5.8) for the potential, it is easy to realize that functions which were appropriate for expansion (2.9) will also be good in the case of expansions (5.7a) and (5.8). Hence in expansion (2.10) we choose the same functions $f$ used in expansion (2.9), namely $f_{j}(p)$ given by Eq. (5.5).

Finally, we consider approximations (2.13) and (2.18). In Eq. (2.13), $\left\langle q|V| f_{i}\right\rangle$ and $\left\langle g_{j} \mid p\right\rangle$ should be good functions to represent the $q$ and $p$ dependences of $\langle q|V| p\rangle$, respectively. This can be easily achieved if we choose $f$ and $g$ to be given by (5.5) and (5.6), respectively. Recalling that Eq. (2.18) results if we substitute the approximate $t$ matrix given by $(2.13 a)$ on the righthand side of the LS equation $T=V+T G_{0} V$, it is easy to realize that $f$ and $g$ given by (5.5) and (5.6) are appropriate choices in (2.18).

Though we shall not present in this paper numerical results for other degenerate kernel schemes presented in Sec. II it is now easy to choose appropriate functions for other methods. For example, approximations (2.15) and (2.19) will present good convergence if we take all the expansion functions $g$ in these methods to be given by (5.6).

After having decided about the expansion functions, we are now prepared to perform numerical calculations with approximations (2.9), (2.10), (2.13), and (2.18). Though we have chosen the functional form of the expansion functions, there is still some arbitrariness left in the choice of the parameter $\beta$ in (5.2) and (5.5). This arbitrariness is turned to good advantage - we vary $\beta$ numerically to obtain rapid convergence. Though in the context of each of the above approximations a new $\beta$ would lead to best convergence, we prefer to choose the same $\beta$ in all approximations for the same function. However, $\beta$ is taken to be different if the functions are different; specifically, we choose one $\beta$ for Eq. (5.6) and another $\beta$ for Eq. (5.5) in order to obtain best convergence.

We performed numerical calculation in momentum space in double precision-correct up to 14 significant figures. An explicit momentum space representation of the quantities involved in numerical calculation can be obtained in Refs. 7 and 11. After a little experimentation we fixed $\beta=3.5 \mathrm{fm}^{-1}$ in Eq. (5.6) and $\beta=2.5 \mathrm{fm}^{-1}$ in Eq. (5.5). In all four cases final convergence was impressive, but the results were not very good for small $N$. By making a change of basis functions we can make certain rank-1 $(N=1) t$ matrix elements exact if we modify the first function(s) in a set following Refs. 7 and 11, and this will significantly improve the convergence rate for small $N$.

In the case of expansion (2.9), the new basis functions $\bar{f}_{i}$ are defined by ${ }^{7}$

$$
\begin{aligned}
& V\left|\bar{f}_{1}\right\rangle=T|p\rangle, \\
& \left|\bar{f}_{i}\right\rangle=\left|f_{i}\right\rangle, \quad i=2,3, \ldots .
\end{aligned}
$$

This new set of expansion functions when used in expansion (2.9) will lead to a rank-N $t$ matrix $T_{N}$ with the properties:

$$
\begin{aligned}
& T_{N}|p\rangle=T|p\rangle, \quad N=1,2, \ldots \\
& \langle p| T_{N}=\langle p| T, \quad N=1,2, \ldots
\end{aligned}
$$

where, of course, we use the modified set $\bar{f}_{i}$ for both $\left|f_{i}\right\rangle$ and its transpose $\left\langle f_{i}\right|$. If we take $p=k$, the onshell momentum at an energy, the half-on-shell $t$ matrix of any rank will be exact.

The practical implementation of (5.9) is easy after we have performed calculations with the old set at a particular energy. In fact, we replace $T$ of $(5.9 \mathrm{a})$ by $T_{N^{\prime}}$ of (2.9), where $N^{\prime}$ is sufficiently large that $T_{N^{\prime}}$ has essentially converged. Then, (5.9a) becomes, for $p=k=0 \mathrm{fm}^{-1}$, the on-shell momentum at zero energy,

$$
V\left|\bar{f}_{1}\right\rangle=\sum_{i, j=1}^{N^{\prime}} V\left|f_{i}\right\rangle D_{i j}\left\langle f_{j}|V| k\right\rangle,
$$

so that

$$
\left|\bar{f}_{1}\right\rangle=\sum_{i=1}^{N^{\prime}}\left|f_{i}\right\rangle C_{i}(k)
$$

with

$$
C_{i}(k)=\sum_{j=1}^{N^{\prime}} D_{i j}\left\langle f_{j}|V| k\right\rangle,
$$

where all the calculations in (5.11) are performed at zero energy. Thus the new basis function $\bar{f}_{1}$ is available as a linear combination of the old functions. In practice, we used $N^{\prime}=10$.

The same thing can be easily done in the case of Eq. (2.13). It is easily seen that in order to satisfy property (5.10) in this case, one must use new basis functions defined by

$$
\begin{aligned}
& V\left|\bar{f}_{1}\right\rangle=T|p\rangle, \\
& \left\langle\bar{g}_{1}\right|=\langle p| T, \\
& \left|\bar{f}_{i}\right\rangle=\left|f_{i}\right\rangle, \quad\left\langle\bar{g}_{i}\right|=\left\langle g_{i}\right|, \quad i=2,3, \ldots .
\end{aligned}
$$

Again, in practical implementation we take $p=k=0 \mathrm{fm}^{-1}$, the on shell momentum at zero energy. Using (2.13) and (5.12), one easily obtains

$$
\begin{aligned}
& \left|\bar{f}_{1}\right\rangle=\sum_{i=1}^{N^{\prime}}\left|f_{i}\right\rangle \hat{C}_{i}(k), \\
& \left\langle\bar{g}_{1}\right|=\sum_{i=1}^{N^{\prime}}\left\langle g_{i}\right| \hat{C}_{i}^{\prime}(k),
\end{aligned}
$$

together with Eq. (5.12c). In Eq. (5.13), 


$$
\begin{aligned}
& \hat{C}_{i}(k)=\sum_{j=1}^{N^{\prime}} D_{i j}\left\langle g_{j} \mid k\right\rangle, \\
& \hat{C}_{i}^{\prime}(k)=\sum_{j=1}^{N^{\prime}}\left\langle k|V| f_{j}\right\rangle D_{j i} .
\end{aligned}
$$

Again, the new basis functions are obtained as a linear combination of the old functions. In practice, we employed $N^{\prime}=10$.

Finally, it is easy to verify that in order to have the property (5.10) in the case of expansion (2.18), one should choose, for $p=k=0^{-1} \mathrm{fm}$,

$$
V\left|\bar{f}_{1}\right\rangle=(T-V)|k\rangle
$$

and

$$
\left\langle\bar{g}_{1}\right| G_{0} V=\langle p|(T-V),
$$

together with (5.12c). The implementation of (5.15) again is not difficult. However, in this case we used the expansion functions given by $(5.12 \mathrm{c})$ and (5.13). With this choice-given by $(5.12)$ - it is easy to verify that only Eq. (5.10b) is satisfied and Eq. (5.10a) is not satisfied. In this case we have a one-sided KowalskiNoyes property.

In case of approximation (2.10) we do not perform any
TABLE I. The coefficients $C, \hat{C}$, and $\hat{C}^{\prime}$ defining the new functions at energy $E=k^{2}=0$. These coefficients are defined by Eqs. (5.11b) and (5.14).

\begin{tabular}{rrrr}
\hline \hline $\mathrm{i}$ & \multicolumn{1}{c}{$C_{i}(k)$} & \multicolumn{1}{c}{$\hat{C}_{i}(k)$} & \multicolumn{1}{c}{$\hat{C}_{i}^{\prime}(k)$} \\
\hline 1 & -36.5758 & -36.3284 & 70.9539 \\
2 & -56.0848 & -56.5445 & 36.0544 \\
3 & 31.1240 & 30.1631 & -81.7214 \\
4 & 28.8955 & 28.7684 & -10.0807 \\
5 & 11.8785 & 12.8540 & 10.5718 \\
6 & -0.7737 & -0.7223 & 12.9460 \\
7 & -6.9354 & -4.7506 & 5.8926 \\
8 & -9.9163 & -7.2262 & 2.4709 \\
9 & 0.2502 & -2.9682 & 0.6873 \\
10 & -0.1465 & -3.1923 & 0.6361 \\
\hline \hline
\end{tabular}

change of expansion functions. This case is particularly more complicated than other examples studied, in that here we need two matrix inversions, whereas in other examples we need only one matrix inversion. Even then we obtain good convergence in this case.

In Table I we present the coefficients $C_{i}$ of (5.11), and $\hat{C}_{i}$ and $\hat{C}_{i}^{\prime}$ of (5.14). The values of $\beta$ used are $\beta=2.5$ $\mathrm{fm}^{-1}$ in Eq. (5.5) and $\beta=3.5 \mathrm{fm}^{-1}$ in Eq. (5.6). In Table II we present the on shell phase shifts at different ener-

TABLE II. Phase shifts for the Reid ${ }^{1} S_{0}$ potential for different $N$ calculated by using the following degenerate kernel schemes: A-Eq. (2.9), B-Eq. (2.13), C-Eq. (2.18), and D-Eq. (2.10). The entries at $E_{\text {c.m. }}=0$ are the scattering lengths in $\mathrm{fm}$. The results for $N=16$ have all converged to four significant figures. The numbers for schemes $\mathrm{A}-\mathrm{C}$ for $N \leq 10$ have been calculated using a modification of the basis function as described in the text.

\begin{tabular}{ccccccccc}
\hline \hline $\begin{array}{c}E_{\text {c.m. }} \\
(\mathrm{MeV})\end{array}$ & & \multicolumn{1}{c}{ Results for $N=$} \\
\hline 0 & A & -17.15 & -17.15 & -17.15 & -17.15 & -17.15 & -17.15 & -17.15 \\
& B & -17.10 & -17.10 & -17.10 & -17.10 & -17.10 & -17.14 & -17.15 \\
& C & -17.12 & -17.12 & -17.12 & -17.12 & -17.12 & -17.14 & -17.15 \\
& D & 0.52 & -14.02 & -15.96 & -17.15 & -17.12 & -17.14 & -17.15 \\
12 & A & 0.8191 & 0.8500 & 0.8554 & 0.8604 & 0.8604 & 0.8605 & 0.8606 \\
& B & 0.8188 & 0.8279 & 0.8467 & 0.8576 & 0.8605 & 0.8606 & 0.8606 \\
& C & 0.5796 & 0.8367 & 0.8545 & 0.8603 & 0.8606 & 0.8606 & 0.8606 \\
& D & -0.2776 & 0.8664 & 0.8378 & 0.8573 & 0.8608 & 0.8606 & 0.8606 \\
48 & A & 0.3705 & 0.4535 & 0.4363 & 0.4398 & 0.4398 & 0.4401 & 0.4402 \\
& B & 0.3707 & 0.4174 & 0.4448 & 0.4417 & 0.4398 & 0.4402 & 0.4402 \\
& C & -0.7158 & 0.4411 & 0.4444 & 0.4403 & 0.4402 & 0.4402 & 0.4402 \\
& D & -0.5412 & 0.4330 & 0.4356 & 0.4403 & 0.4403 & 0.4402 & 0.4402 \\
72 & A & 0.2053 & 0.2783 & 0.2577 & 0.2620 & 0.2624 & 0.2627 & 0.2630 \\
& B & 0.2059 & 0.2596 & 0.2702 & 0.2628 & 0.2626 & 0.2629 & 0.2630 \\
& C & -1.0582 & 0.2624 & 0.2636 & 0.2621 & 0.2628 & 0.2630 & 0.2630 \\
& D & -0.6522 & 0.2558 & 0.2691 & 0.2636 & 0.2636 & 0.2630 & 0.2630 \\
104 & A & 0.0672 & 0.0859 & 0.0758 & 0.0781 & 0.0798 & 0.0802 & 0.0803 \\
& B & 0.0672 & 0.0925 & 0.0831 & 0.0749 & 0.0801 & 0.0803 & 0.0803 \\
& C & -1.2694 & 0.0730 & 0.0795 & 0.0799 & 0.0802 & 0.0803 & 0.0803 \\
& D & -0.7678 & 0.0717 & 0.0911 & 0.0815 & 0.0803 & 0.0805 & 0.0803 \\
176 & A & 0.0299 & -0.2499 & -0.2217 & -0.2167 & -0.2180 & -0.2167 & -0.2164 \\
& B & 0.0303 & -0.2019 & -0.2289 & -0.2240 & -0.2180 & -0.2164 & -0.2164 \\
& C & -1.4458 & -0.2212 & -0.2148 & -0.2183 & -0.2166 & -0.2164 & -0.2164 \\
& D & -0.9561 & -0.2549 & -0.2048 & -0.2253 & -0.2177 & -0.2160 & -0.2164 \\
\hline \hline
\end{tabular}


gies and scattering lengths in these four cases. It is easy to realize that the convergence is much better than that previously obtained in any degenerate kernel method ${ }^{5-15}$ using the Reid ${ }^{1} S_{0}$ potential. For example, it is better than that obtained in Refs. 7, 9, 11, 12, and 14. The off-shell matrix elements also converge equally well, in general.

Unfortunately, we should not compare results of different approximation schemes shown in Table II and claim superiority of one scheme over another because we are using basically the same expansion function in all the cases studied. In order to obtain the best convergence the expansion functions in each scheme are supposed to be different. Specifically, though with a particular choice of expansion function and for a particular potential - the Reid ${ }^{1} S_{0}$ potential - one approximation scheme converges faster than another, the situation may change if the expansion functions and the potential are changed. The schemes (2.9), (2.10), and (2.13) can all be derived from Schwinger-type variational principles and will yield identical results if the expansion functions used in the scheme are related in a certain way. For example, Eqs. (2.9a) and (2.13a) will yield identical results if $\left\langle f_{j}\right|$ of $(2.9 \mathrm{a})$ is related to $\left\langle g_{j}\right|$ of $(2.13 \mathrm{a})$ by $\left\langle f_{j}\right| V=\left\langle g_{j}\right|$. In other words, expansions (2.9), (2.10), and (2.13) are all equivalent. Expansion (2.18) can be derived from a variational principle of the Hulthén-Kohn type and is not related to a variational principle of the Schwinger type. Also, as has been pointed out before, one should not claim the superiority of one type of variation principle over another, because such a claim will be limited, in general, to a special case and will depend on the expansion functions and the potential used.

\section{SUMMARY}

We have presented a unified formulation of the degenerate kernel scheme and the method of separable expansions for solving LS-type equations, and have established a relation between them. We have shown that all degenerate kernel schemes can be derived from a variational principle. We have proposed various practical methods for solving LS-type equations. We have performed numerical calculations - for some of the schemes - in the case of the Reid ${ }^{1} S_{0}$ potential. It is pointed out that if the expansion functions are appropriately chosen, good convergence can be obtained in each of these cases. The numerical convergence obtained in the present case is better than that obtained previously in the case of the Reid ${ }^{1} S_{0}$ potential. A correct and appropriate guess of expansion functions is crucial for a quick convergence.

Though we have considered the application of the present approach to the solution of the LS equation with the Reid potential, the present approach should find interesting application in other areas of physics in the solution of Fredholm integral equations of the second kind. Interesting applications of similar approaches in the area of atomic physics are to be found in Refs. 3, 6, and 22. The last article ${ }^{22}$ is particularly interesting, wherein the authors solve, for the first time, four-particle scattering equations, which are integral equations of the Fredholm type, for the atom-triatom system using the separable expansion technique of Ref. 18.

This work was supported in part by the Financiadora de Estudos e Projetos (FINEP) and the Conselho Nacional de Desenvolvimento Científico e Tecnológico (CNPq) of Brazil.
${ }^{1}$ J. Schwinger, lecture notes, Harvard University, 1947 (unpublished).

${ }^{2}$ W. Kohn, Phys. Rev. 74, 1763 (1948); L. Hulthén, Kgl. Fysiograf. Sallskap. und Forh. 14, 257 (1944); Ark. Mat. Astron. Fys. 35A, No. 25 (1948).

${ }^{3}$ For reviews on variational principles, see J. Callaway, Phys. Rep. 45, 89 (1978); M. A. Abdel-Raouf, ibid. 84, 163 (1982); 108, 1 (1984); L. M. Delves, Adv. Nucl. Phys. 5, 1 (1972); D. G. Truhlar, J. Abdallah, Jr., and R. L. Smith, Adv. Chem. Phys. 25, 211 (1974); R. K. Nesbet, Adv. At. Mol. Phys. 13, 315 (1979); E. Gerjuoy, A.R.P. Rau, and L. Spruch, Rev. Mod. Phys. 55, 725 (1983).

${ }^{4}$ E. Gerjuoy and D. S. Saxon, Phys. Rev. 94, 478 (1954); M. Moe and D.S. Saxon, ibid. 111, 950 (1958).

${ }^{5}$ I. H. Sloan and T. J. Brady, Phys. Rev. C 6, 701 (1972).

${ }^{6}$ K. Takatsuka, R. R. Lucchese, and V. McKoy, Phys. Rev. A 24, 1812 (1981); J. Callaway, Phys. Lett. 77A, 137 (1980); K. Takatsuka and V. McKoy, Phys. Rev. A 23, 2352 (1981).

${ }^{7}$ S. K. Adhikari and I. H. Sloan, Phys. Rev. C 11, 1133 (1975); S.K. Adhikari, ibid. 10, 1623 (1974).

${ }^{8}$ B. A. Lippmann and J. Schwinger, Phys. Rev. 79, 469 (1950).

${ }^{9}$ R. Weinberg, Phys. Rev. 131, 440 (1963); M. G. Fuda, ibid. 186, 1078 (1969); E. Harms, Phys. Rev. C 1, 1667 (1970).

${ }^{10}$ D. J. Ernst, C. M. Shakin, and R. M. Thaler, Phys. Rev. C 8,
46 (1973); H. Bateman, Proc. R. Soc. London, Ser. A 100, 441 (1922).

${ }^{11}$ S. K. Adhikari and I. H. Sloan, Nucl. Phys. A241, 429 (1975). ${ }^{12}$ I.H. Sloan and S. K. Adhikari, Nucl. Phys. A235, 352 (1974).

${ }^{13}$ S. Oryu, Phys. Rev. C 27, 2500 (1983).

14J. Révai, M. Sotona, and J. Žofka, J. Phys. G 11, 745 (1985).

${ }^{15}$ G. H. Rawitscher and G. Delic, Phys. Rev. C 29, 747 (1984); G. H. Rawitscher, ibid. 25, 2196 (1982).

${ }^{16}$ K. L. Kowalski, Phys. Rev. Lett. 15, 798 (1965); H. P. Noyes, ibid. 15, 538 (1965).

${ }^{17}$ C. Lovelace, Phys. Rev. 135, B1225 (1964); R. D. Amado, ibid. 132, 485 (1963); A. N. Mitra, Nucl. Phys. 32, 529 (1962).

${ }^{18}$ S. Sofianos, N. J. McGurk, and H. Fiedeldey, Nucl. Phys. A318, 295 (1979); A. Casel, H. Haberzettl, and W. Sandhas, Phys. Rev. C 25, 1738 (1982); A. C. Fonseca, H. Haberzettl, and E. Cravo, ibid. 27, 939 (1983).

${ }^{19}$ R. V. Reid, Jr., Ann. Phys. (N.Y.) 50, 411 (1968).

${ }^{20}$ M. A. Abdel-Raouf, Phys. Rev. A 30, 2794 (1984).

${ }^{21}$ Higher Transcendental Functions, edited by A. Erdélyi (McGraw-Hill, New York, 1953), Vol. I.

${ }^{22}$ A. C. Fonseca and T.K. Lim, Phys. Rev. Lett. 55, 1285 (1985). 